\title{
POSSIBLE DETECTION OF COLLIDING PLASMOIDS IN THE TAIL OF COMET KOHOUTEK (1973f)
}

\author{
R.G. Roosen and J.C. Brandt
}

\begin{abstract}
Six JOCR photographs of Comet Kohoutek taken on January 19, 1974 (UT) show concentrations of plasma about ten degrees from the head of the comet which are very similar to the "barred spirals" reported by Bostick (IAU Symposium No. 6: Electromagnetic Phenomena in Cosmical Physics, B. Lehnert, (ed.) Cambridge Univ. Press, 1958) from laboratory observations of colliding plasmoids. The photographs were taken about one day after a solar magnetic sector boundary crossed the comet's head. Also, the comet's plasma tail splits near the head into two distinct segments. It appears that the two segments reconverge and form the plasma concentrations by collisions. This represents an increase in scale over Bostick's work of about $10^{14}$ Possible historical observations of similar phenomena are discussed.
\end{abstract}




\section{DISCUSSION}

G. H. Herbig: Can it be that this object is due to an internal reflection in your optical system? In Schmidt systems, reflection from the emulsion (or filter, or field flattener) back through the system and off the correcting lens and thus to the focal plane again can produce an out-of-focus image of a bright star. These ghosts are symmetrical with respect to the field center. I notice that on your first slide the comet head is located with respect to the lower left corner just as your feature is placed with respect to the upper right.

R. G. Roosen: First of all, we took plates with and without a filter, so if it were reflection, the reflection would have to be the same both with and without a filter. That's a fine suggestion, and I'll be happy to embarrassingly publish a retraction if it turn's out that that's it, and I will go back and measure the plates for this distance.

F. L. Whipple: I hate to be the "devil's advocate," but in tens of thousands of plate inspections I have found so many false images that I have a built-in suspicion of peculiarly shaped images that move for a short duration.

R. G. Roosen: We've examined all our other plates and don't find anything like this on any of the other nights. Klauss Jockers said that he thought that he found one on another plate (he's examined all of our plates, too), but we went to look for it and couldn't find it. So on this night it showed up on every plate, and it never showed up on any of the other nights, even though we had this same orientation.

P. M. Millman: In regard to cross-axis inverted images, these do not necessarily depend on the closeness of the original image and the cross-axis image to the edge of the plate. The important fact is that the two images should be the same angular distance from the optical axis of the lens system.

B. G. Marsden: Peculiar tail features of this kind have been reported before, of course, and I mention in particular one appearance in a photograph of Comet Bennett by McClure. The fact that your feature also appears on a red plate makes one wonder whether some of the cometary objects occasionally reported visually near bright comets are perhaps manifestations of the same phenomenon. Barnard is said to have dreamt one night, during his comet-hunting period, that the sky was filled with comets. On waking and going outdoors, he found that it was, with a dozen or so "comets" seen to be surrounding the great comet of 1882. Similar objects were also reported by Brooks and Schmidt. 